\title{
Remdesivir Induced Liver Injury and Severe COVID-19 Infection
}

\author{
Chanchal Kumar Ghosh", S. M. Ali Hasan, Suman Dey \\ Department of Gastroenterology, Bangabandhu Sheikh Mujib Medical University, Dhaka, Bangladesh
}

Email address:

dr.chanchalghosh $a$ gmail.com (C. K. Ghosh)

${ }^{*}$ Corresponding author

\section{To cite this article:}

Chanchal Kumar Ghosh, S. M. Ali Hasan, Suman Dey. Remdesivir Induced Liver Injury and Severe COVID-19 Infection. American Journal of Internal Medicine. Vol. 8, No. 6, 2020, pp. 285-288. doi: 10.11648/j.ajim.20200806.18

Received: November 4, 2020; Accepted: November 13, 2020; Published: November 23, 2020

\begin{abstract}
Background and Aims: Remdesivir is identified as an effective therapeutic option in COVID-19, but its' hepatic safety has not been well studied. So, we aimed to identify the pattern and severity of hepatotoxicity in remdesivir treated COVID-19 patients. Methods: This cross-sectional study was carried out at a dedicated COVID-19 unit of a university hospital in Dhaka, Bangladesh among severe COVID-19 cases. Alterations of liver functions were compared between the remdesivir and the non-remdesivir treated patients. Results: Out of 50 severe COVID-19 cases 25 had received remdesivir and 25 had received other supportive care without remdesivir. Median serum aspartate aminotransferase (AST) and alanine aminotransferase (ALT) values were significantly higher in the remdesivir treated arm (p-value for AST $<0.0001$ and ALT $<0.001$ ). Grade-2 elevation of AST and ALT and grade-3 elevation of AST levels were significantly higher among the remdesivir treated group. No patients had significant bilirubin elevation $(\geq 2.5 \mathrm{mg} / \mathrm{dl})$ and only 1 patient had INR $>1.5$ in the remdesivir treated arm. Conclusion: Many of the patients with severe COVID-19 had mild to moderate aminotransferases elevation. If the elevation of liver enzymes occurs after the initiation of remdesivir, adverse drug reactions need to be considered and drug discontinuation may require if severe elevation occurs.
\end{abstract}

Keywords: Remdesivir, COVID-19, Hepatotoxicity, Aminotransferase

\section{Introduction}

At the end of 2019, a severe acute respiratory syndrome coronavirus 2 (SARS-CoV-2) was first identified in Wuhan city, China results in a respiratory illness designated as coronavirus disease 2019 (Covid-19) [1]. It was initially viewed as primarily a respiratory disease, but is now considered as a complex multisystem disease [2]. SARS-CoV-2-induced hepatic injury has been well described $[3,4]$. So, to evaluate the hepatic safety of drugs administered and to monitor the liver function of COVID-19 patients is very important. Remdesivir, a nucleoside analog, an inhibitor of the viral RNA-dependent, RNA polymerase $[5,6]$ has been identified as a promising therapeutic option for Covid-19 because it inhibits SARS-CoV-2 in vitro [7]. Available data suggest remdesivir has some clinical benefit, although the overall evidence is low [8]. However, the hepatic safety of remdesivir in COVID-19 has not been well studied. So, we aimed to conduct a study to find out the patterns and severity of liver injury in COVID-19 patients treated with remdesivir.

\section{Methods}

This is a cross-sectional study carried out at a dedicated COVID-19 unit of a university hospital in Dhaka, Bangladesh. The study period was July 2020 to September 2020. Fifty RT-PCR positive severe COVID-19 cases aged $\geq 18$ years were recruited according to case definition. Severe COVID-19 was defined as radiological findings of pneumonia in the chest $\mathrm{x}$-ray or HRCT of the chest and any one of the following criteria - a) respiratory rate $\geq 30$ breaths $/ \mathrm{min}$, b) $\mathrm{O}_{2}$ saturation $\left(\mathrm{SpO}_{2}\right) \leq 93 \%$ at rest on the pulse oximeter, c) arterial partial pressure of oxygen $\left(\mathrm{PaO}_{2}\right)$ /fraction of inspires oxygen $\left(\mathrm{FiO}_{2}\right)$ $\leq 300 \mathrm{mmHg}$ in $\mathrm{ABG}$ or $\mathrm{SpO}_{2} / \mathrm{FiO}_{2}<315 \mathrm{mmHg}$ [9]. Severe COVID-19 cases were grouped into two groups according to those who had received at least 5 days of i.v remdesivir (200 
mg loading dose on day 1 , followed by $100 \mathrm{mg}$ daily for up 4 additional days). Impairments of hepatic functions were compared between the remdesivir and the non-remdesivir treated group.

Patients were assessed clinically and with laboratory parameters. Aminotransferase elevation was graded as grade-1 (1.25 to 3-fold elevation), grade-2 (3 to 5-fold elevation) and grade-3 (more than 5-fold elevation). Patients with preexisting liver disease, positive serology for the viral marker, and known exposure to indigenous drugs were excluded from the study.

Statistical Analysis

Statistical analyses were performed using IBM SPSS version 25 (IBM Corp., Armonk, NY, USA). Continuous variables were presented as mean and $\mathrm{SD}$, while categorical variables were presented as frequency and percentage. Chi-square test, independent samples t-test, and Mann Whitney U test was conducted for categorical, normal, and skewed continuous variables respectively. A P-value of $<0.05$ was considered to be statistically significant.

The study was approved by the University Institutional Ethical Review Board. Informed consent was obtained from each patient in the study.

\section{Result}

A total of 50 severe COVID-19 cases was included in this study. Of them, 25 had received remdesivir along with other supportive care and 25 had received other supportive care without remdesivir. The mean age was $57.3 \pm 13.3$ and $55.6 \pm 12.7$ years among remdesivir and non-remdesivir groups respectively. Male (68\%) were predominant in both groups. There was no significant difference in mean body mass index (BMI) and Oxygen saturation $\left(\mathrm{SpO}_{2}\right)$ level among both groups Baseline characteristics are shown in table 1.

Table 1. Baseline characteristics of the study population.

\begin{tabular}{llll}
\hline & Remdesivir treated group (n=25) & Non-remdesivir treated group (n=25) & P-value \\
\hline Age $($ mean \pm SD) & $57.3 \pm 13.2$ & $55.6 \pm 12.7$ & 0.641 \\
Gender (Male) & $17(68.0 \%)$ & $17(68.0 \%)$ & 1.000 \\
BMI (mean \pm SD) & $23.4 \pm 3.9$ & $23.6 \pm 2.8$ & 0.794 \\
Obesity & $2(8.0 \%)$ & $1(4.0 \%)$ & 1.000 \\
$\mathrm{O}_{2}$ saturation $(\operatorname{mean} \pm \mathrm{SD})$ & $87.0 \pm 3.8$ & $87.1 \pm 5.7$ & 0.907 \\
\hline
\end{tabular}

Values are presented as mean $\pm \mathrm{SD}$ and number (percentage).

Median serum bilirubin, prothrombin time, INR, and serum albumin values were similar in both groups. Serum aspartate aminotransferase (AST) and alanine aminotransferase (ALT) values were significantly higher in the remdesivir treated arm ( $\mathrm{p}$-value for AST $<0.0001$ and ALT $<0.001$ ). Median serum alkaline phosphatase (ALP) value was also significantly higher in the remdesivir treated group (p-value 0.005) but within the upper limit of the normal range. No patients had bilirubin elevation of $\geq 2.5 \mathrm{mg} / \mathrm{dl}$ and only 1 patient had INR $>1.5$ in the remdesivir treated arm. Laboratory parameters of the study participants are shown in table 2 .

Table 2. Laboratory parameters of the study population.

\begin{tabular}{llll}
\hline & Remdesivir treated group $(\mathbf{n}=\mathbf{2 5})$ & Non-remdesivir treated group $(\mathbf{n}=\mathbf{2 5})$ & P-value \\
\hline S. Bilirubin $(\mathrm{mg} / \mathrm{dl})$ & $0.6(0.15-2.4)$ & $0.6(0.3-2.3)$ & 0.907 \\
AST $(\mathrm{u} / \mathrm{L})$ & $120(35-275)$ & $46(17-149)$ & $<0.0001$ \\
ALT $(\mathrm{u} / \mathrm{L})$ & $104(27-276)$ & $38(14-243)$ & 0.001 \\
Alkaline phosphatase $(\mathrm{u} / \mathrm{L})$ & $129(51-371)$ & $88(57-230)$ & 0.005 \\
Prothrombin time $(\mathrm{sec})$ & $13.1(12.0-22.0)$ & $13.4(12.0-17.2)$ & 0.586 \\
INR & $1.10(1.0-1.9)$ & $1.03(1.0-1.4)$ & 0.411 \\
S. Albumin & $35.6 \pm 5.8$ & $35.1 \pm 3.5$ & 0.747 \\
\hline
\end{tabular}

Values are presented as median (range) and mean \pm SD. AST, Aspartate aminotransferase; ALT, Alanine aminotransferase; INR, International normalization ratio.

Though higher numbers of grade-1 elevation of AST and ALT were observed in remdesivir treated arm in comparison to non-remdesivir treated arm $(44.0 \%$ vs. $32 \%$ and $40.0 \%$ vs. $24.0 \%$ respectively), the difference is not statistically significant. Remdesivir treated group had a significantly higher frequency of grade-2 AST and ALT elevation in comparison to the non-remdesivir treated group (36.0\% vs. $12 \%$ and $40.0 \%$ vs. $8.0 \%$ respectively). The odds ratio for the grade-2 elevation of AST level in the remdesivir treated group was 1.78 with a $95 \%$ confidence interval (CI) 1.09 to 2.92. The odds ratio for the grade- 2 elevation of ALT level in the remdesivir treated group was 2.11 with a 95\% CI 1.32 to 3.37. All patients with grade-3 elevation of AST (16.0\%) were in the remdesivir treated group, the odds ratio was 2.19 with a $95 \%$ CI 1.60 to 3.00 . Aminotransferase elevation in the remdesivir and the non-remdesivir treated group is shown in tables 3-4. 
Table 3. Aspartate aminotransferase (AST) elevation between remdesivir and non-remdesivir treated group.

\begin{tabular}{lllll}
\hline AST elevation & Treatment group & Number (\%) & Odds ratio & 95\% CI \\
\hline \multirow{2}{*}{ Grade-1 } & Remdesivir group & $11(44.0 \%)$ & 1.28 & $0.74-2.21$ \\
& Non-remdesivir group & $8(32.0 \%)$ & 0.77 & $0.41-1.42$ \\
Grade-2 & Remdesivir group & $9(36.0 \%)$ & 1.78 & $1.09-2.92$ \\
& Non-remdesivir group & $3(12.0 \%)$ & 0.43 & $0.16-1.19$ \\
\multirow{2}{*}{ Grade-3 } & Remdesivir group & $4(16.0 \%)$ & 2.19 & 0.047 \\
& Non-remdesivir group & $0(0.0 \%)$ & & 0.05 \\
\hline
\end{tabular}

AST, Aspartate aminotransferase; CI, Confidence interval.

Table 4. Alanine aminotransferase (ALT) elevation between remdesivir and non-remdesivir treated group.

\begin{tabular}{lllll}
\hline ALT elevation & Treatment group & Number (\%) & Odds ratio & 95\% CI \\
\hline \multirow{2}{*}{ Grade-1 } & Remdesivir group & $10(40.0 \%)$ & 1.42 & $0.83-2.42$ \\
& Non-remdesivir group & $6(24.0 \%)$ & 0.67 & $0.33-1.35$ \\
Grade-2 & Remdesivir group & $10(40.0 \%)$ & 2.11 & $1.32-3.37$ \\
& Non-remdesivir group & $2(8.0 \%)$ & 0.28 & 0.225 \\
\multirow{2}{*}{ Grade-3 } & Remdesivir group & $1(4.0 \%)$ & 1.00 & $0.08-1.00$ \\
& Non-remdesivir group & $1(4.0 \%)$ & 1.00 & 0.008 \\
\hline
\end{tabular}

ALT, Alanine aminotransferase; CI, Confidence interval.

\section{Discussion}

SARS-COV2 infection can cause an increase in liver enzymes in $14-53 \%$ of cases [4, 10-12]. The mechanisms are mostly unknown. It may be due to virus-induced inflammation, hypoxic liver injury, and/or drug-induced liver injury. Among several drugs used for the treatment of COVID-19, remdesivir is one of the promising drugs. Information on the safety profile of remdesivir is rapidly evolving. Several clinical studies reported aminotransferase elevations following remdesivir treatment [13-15], which is consistent with our findings. In our study, remdesivir caused frequent grade-1 and grade-2 elevation of aminotransferases which didn't require drug discontinuation. It is unclear if the liver function abnormalities observed in patients with COVID-19 treated with remdesivir are due to the infectious process or the drug itself. Although Wang et al, and the ACTT-1 study reported that, aminotransferases elevation were infrequent and occurred in similar proportions of remdesivir and placebo-treated patients $[13,16]$, we observed significantly higher numbers of grade- 2 and grade-3 aminotransferases elevation in remdesivir treated arm compared to the non-remdesivir treated arm. Transient mild ALT elevations were reported in most subjects in the multi-dose PK studies, including one individual with ALT values more than 10 times from baseline [17]. All three patients out of the first 12 COVID-19 cases in the US who received remdesivir experienced transient aminotransferases elevations [18]. According to Grein et al.'s study on compassionate-use remdesivir against COVID-19, 23\% of the patients reported increased hepatic enzymes, and two of them therefore discontinued remdesivir prematurely [17]. Lescure et al. also reported one COVID-19 patient discontinued remdesivir because of alanine aminotransferase elevation and rash, which then decreased within 3 days [19]. Except for 1 case of hyperbilirubinemia, all other cases were asymptomatic [17]. We found no cases of bilirubin elevation above $2.5 \mathrm{mg} / \mathrm{dl}$. The FDA reported grade 3 and 4 aminotransferase elevations occurred in $7 \%$ and bilirubin elevations were uncommon (1.3\%) with 5- and 10-day courses of remdesivir in patients with COVID-19 [17]. At this time, it is also unclear how remdesivir causes hepatic impairment. It may cause liver injury like other nucleosides. Nucleoside analogs are known to cause liver injury by a variety of mechanisms [20]. Mitochondrial dysfunction caused by inhibition of mitochondrial DNA synthesis is the most commonly involved mechanism. This can affect various tissues, leading to neuropathy, myopathy, pancreatitis, marrow suppression, and/or liver injury [20]. To date, no extra-hepatic manifestations of mitochondrial dysfunction have been reported in patients treated with remdesivir. Other mechanisms may include acute hypersensitivity reactions or the production of toxic intermediates to cause liver injury [20].

\section{Conclusion}

Since many of the patients with severe COVID-19 had mild to moderate aminotransferases elevation, if the elevation of liver enzymes occurs after the initiation of remdesivir, adverse drug reactions need to be considered. Drug discontinuation may require if severe elevation occurs. Although it is reassuring that, most of the cases are non-severe and asymptomatic, it is unknown that asymptomatic abnormalities are harbingers of more serious liver injury. Remdesivir should not be used with other hepatotoxic drugs or in preexisting liver disease. The monitoring of the liver function is warranted during the treatment.

\section{References}

[1] Helmy YA, Fawzy M, Elaswad A, Sobieh A, Kenney SP, Shehata AA. The COVID-19 Pandemic: A Comprehensive Review of Taxonomy, Genetics, Epidemiology, Diagnosis, Treatment, and Control. J Clin Med. 2020; 9 (4): 1225. 
[2] Roberts CM, Levi M, McKee M, Schilling R, Lim WS, Grocott MPW. COVID-19: a complex multisystem disorder. $\mathrm{Br} J$ Anaesth. 2020; 125 (3): 238-242.

[3] Zhao D, Yao F, Wang L, et al. A Comparative Study on the Clinical Features of Coronavirus 2019 (COVID-19) Pneumonia with Other Pneumonias. Clin Infect Dis. 2020; 71 (15): 756-761.

[4] Xu L, Liu J, Lu M, Yang D, Zheng X. Liver injury during highly pathogenic human coronavirus infections. Liver Int. 2020; 40 (5): 998-1004.

[5] Agostini ML, Andres EL, Sims AC, et al. Coronavirus Susceptibility to the Antiviral Remdesivir (GS-5734) Is Mediated by the Viral Polymerase and the Proofreading Exoribonuclease. mBio. 2018; 9 (2): e00221-00218.

[6] Brown AJ, Won JJ, Graham RL, et al. Broad spectrum antiviral remdesivir inhibits human endemic and zoonotic deltacoronaviruses with a highly divergent RNA dependent RNA polymerase. Antiviral Res. 2019; 169: 104541.

[7] Wang M, Cao R, Zhang L, et al. Remdesivir and chloroquine effectively inhibit the recently emerged novel coronavirus (2019-nCoV) in vitro. Cell Research. 2020; 30 (3): 269-271.

[8] Rochwerg B, Agarwal A, Zeng L, et al. Remdesivir for severe covid-19: a clinical practice guideline. BMJ (Clinical research ed). 2020; 370: m2924. doi: 10.1136/bmj.m2924. Accessed $2020 / 07 / /$.

[9] National Guidelines on Clinical Management of Coronavirus Disease 2019 (COVID-19). In: Disease Control Division DGoHS, Ministry of Health \& Family Welfare, Government of the People's Republic of Bangladesh, ed. Version 7.0 ed2020.

[10] Huang C, Wang Y, Li X, et al. Clinical features of patients infected with 2019 novel coronavirus in Wuhan, China. Lancet (London, England). 2020; 395 (10223): 497-506.
[11] Guan W-j, Ni Z-y, Hu Y, et al. Clinical Characteristics of Coronavirus Disease 2019 in China. New England Journal of Medicine. 2020; 382 (18): 1708-1720.

[12] Zhang C, Shi L, Wang F-S. Liver injury in COVID-19: management and challenges. The Lancet Gastroenterology \& Hepatology. 2020; 5 (5): 428-430.

[13] Wang Y, Zhang D, Du G, et al. Remdesivir in adults with severe COVID-19: a randomised, double-blind, placebo-controlled, multicentre trial. The Lancet. 2020.

[14] Goldman JD, Lye DCB, Hui DS, et al. Remdesivir for 5 or 10 Days in Patients with Severe Covid-19. New England Journal of Medicine. 2020.

[15] Zampino R, Mele F, Florio LL, et al. Liver injury in remdesivir-treated COVID-19 patients. Hepatol Int. 2020; 14 (5): 881-883.

[16] Beigel JH, Tomashek KM, Dodd LE, et al. Remdesivir for the Treatment of Covid-19 - Final Report. N Engl J Med. 2020.

[17] Grein J, Ohmagari N, Shin D, et al. Compassionate Use of Remdesivir for Patients with Severe Covid-19. N Engl J Med. 2020; 382 (24): 2327-2336.

[18] Kujawski SA, Wong KK, Collins JP, et al. Clinical and virologic characteristics of the first 12 patients with coronavirus disease 2019 (COVID-19) in the United States. Nature Medicine. 2020.

[19] Lescure F-X, Bouadma L, Nguyen D, et al. Clinical and virological data of the first cases of COVID-19 in Europe: a case series. The Lancet Infectious Diseases. 2020.

[20] Munster VJ, Feldmann F, Williamson BN, et al. Respiratory disease and virus shedding in rhesus macaques inoculated with SARS-CoV-2. BioRxiv. 2020. 